\title{
Antenatal Corticosteroids Prior to 24 Weeks' Gestation and Neonatal Outcome of Extremely Low Birth Weight Infants
}

\author{
Soraya Abbasi, M.D., ${ }^{1}$ Corrina Oxford, M.D., ${ }^{2}$ Jeffrey Gerdes, M.D., ${ }^{1}$ \\ Harish Sehdev, M.D., ${ }^{2}$ and Jack Ludmir, M.D. ${ }^{2}$
}

The publisher regrets an error in the table titles of Tables 1 and 2 in the above article in the American Journal of Perinatology, Volume 27, Number 1, 2010, p. 63. The correct tables appear below.

Table 1 Maternal and Delivery Characteristics of Singleton Pregnancies Delivered at 23\% to $25^{6} / 7$ Weeks' Gestation

\begin{tabular}{llll}
\hline Characteristics & Steroid-Treated $(\boldsymbol{n}=\mathbf{8 2})$ & Controls $(\boldsymbol{n}=\mathbf{3 5})$ & $\boldsymbol{p}$ Value \\
\hline Maternal age (y) & $28.7 \pm 7.1$ & $26.9 \pm 7.2$ & 0.22 \\
Black/white/other (\%) & $65 / 23 / 13$ & $66 / 17 / 17$ & 0.90 \\
Preeclampsia (\%) & 9.8 & 2.9 & 0.37 \\
Preterm labor (\%) & 77.8 & 68.9 & 0.61 \\
Chorioamnionitis (\%) & 76.8 & 54.3 & 0.03 \\
Premature rupture of membranes (\%) & 59.8 & 34.3 & 0.02 \\
Cesarean section (\%) & 47.6 & 40.0 & 0.58 \\
Gestational age at ANS dose (wk) & $23.5 \pm 0.5$ & & \\
\hline
\end{tabular}

Data expressed as percentage and mean \pm standard deviation. ANS, antenatal corticosteroid.

\footnotetext{
${ }^{1}$ Department of Pediatrics, University of Pennsylvania, Philadelphia, Pennsylvania; ${ }^{2}$ Department of Obstetrics and Gynecology, University of Pennsylvania, Philadelphia, Pennsylvania.

Address for correspondence and reprint requests: Soraya Abbasi, M.D., CHOP Newborn Care, Pennsylvania Hospital, 800 Spruce Street, Philadelphia, PA 19107 (e-mail: Soraya.abbasi@uphs.upenn.edu).
}

Am J Perinatol 2011;28:87-88. Copyright (C) 2011 by Thieme Medical Publishers, Inc., 333 Seventh Avenue, New York, NY 10001, USA. Tel: +1(212) 584-4662.

DOI: http://dx.doi.org/10.1055/s-0030-1268900.

ISSN 0735-1631. 
Table 2 Antenatal Corticosteroid Exposure: Outcome of Singleton Infants at 23\% to $25 \% / 7$ Weeks' Gestation

\begin{tabular}{|c|c|c|c|}
\hline Characteristics & Steroid-Treated $(n=82)$ & Controls $(n=35)$ & $p$ Value \\
\hline Birth weight (g) & $702 \pm 165$ & $657 \pm 150$ & 0.14 \\
\hline Gestational age (wk) & $24.3 \pm 1.0$ & $24.1 \pm 1.1$ & 0.30 \\
\hline Sex (\% male/female) & $54.9 / 45.1$ & $57.1 / 42.9$ & 0.84 \\
\hline 1-min Apgar <7 (\%) & 68.3 & 88.6 & 0.02 \\
\hline 5-min Apgar <7 (\%) & 30.5 & 51.4 & 0.03 \\
\hline Death before discharge (\%) & 29.3 & 62.9 & $<0.001$ \\
\hline IVH (grade 1 and 2, \%) & 45.5 & 43.5 & 0.99 \\
\hline IVH (grade 3 and 4, \%) & 16.7 & 36.0 & $<0.05$ \\
\hline $\operatorname{RDS}(\%)$ & 100 & 100 & NA \\
\hline PDA treated with indomethacin (\%) & 50.7 & 72.0 & 0.07 \\
\hline Sepsis (\%) & 60.7 & 52.2 & 0.48 \\
\hline NEC stage 3 and $4(\%)$ & 0.0 & 6.9 & 0.57 \\
\hline Pressors (\%) & 54.4 & 64.5 & 0.35 \\
\hline ROP (stage 3-4) & 32.7 & 23.1 & 0.74 \\
\hline BPD at 36 weeks' PCA (\%) & 71.9 & 76.9 & 0.99 \\
\hline Duration of vent (d) & $51 \pm 37$ & $49 \pm 21$ & 0.80 \\
\hline Duration of $\mathrm{O}_{2}(\mathrm{~d})$ & $55 \pm 38$ & $53 \pm 38$ & 0.72 \\
\hline Length of stay (d) & $117 \pm 52$ & $108 \pm 24$ & 0.41 \\
\hline
\end{tabular}

Data expressed as percentage and mean \pm SD.

PDA, patent ductus arteriosus; NEC, necrotizing enterocolitis; IVH, intraventricular hemorrhage; ROP, retinopathy of prematurity; BPD, bronchopulmonary dysplasia; PCA, postconceptional age. 